\title{
DETERMINATION OF FULLY POSITIVE O.D.F.s IN THE FRAME OF THE HARMONIC METHOD
}

\author{
F. WAGNER \\ LM2P, UFR MIM, Ile du Saulcy, 57045 Metz-Cedex, France
}

(Received 15 February 1991)

Because of the experimental errors in the pole figures the iterative harmonic method does not succeed in every case in getting a fully positive solution $f(g)$. In this paper, it is proposed to apply, in such cases, a final correction in order to ensure the positivity of the ODF.

KEY WORDS ODF analysis, Iterative positivity method, Final positivity correction.

\section{INTRODUCTION}

An elegant way to determine a complete O.D.F. in the frame of the harmonic method is the iterative positivity technique (Dahms and Bunge, 1988; Dahms and Bunge, 1989; Wagner and Dahms, 1991). An iteration is based on the following formula:

$$
f_{p+1}(g)=f_{p}(g)+\lambda_{p+1} \tilde{\hat{x}}_{p+1}(g)
$$

with $f_{p}(g)$ the calculated solution after $p$ steps with

$$
\hat{f}_{p+1}(g)=\left\{\begin{array}{lll}
0 & \text { if } & f_{p}(g) \geqslant 0 \\
-f_{p}(g) & \text { if } & f_{p}(g)<0
\end{array}\right.
$$

and with $\lambda_{p+1}$ an optimization parameter (Wagner et al., 1990). Because the starting solution reads $f_{o}(g)=\tilde{f}(g)(\tilde{f}(g)$ being calculated from pole figures) it is clear from (1) that odd functions are added to this initial solution intending to ensure the positivity after a sufficient number of steps. This method consists then in searching an O.D.F. with a given minimum (it is fixed to 0 . in formula (2) but can be taken as a positive value $r_{\text {min }}$ (Dahms and Bunge, 1988; Wagner et al., 1991)). Among the possible solutions, the closest one to the initial solution $f_{o}(g)$ will be chosen by the algorithm (Wagner et al., 1990). Tests with model functions (i.e. pole figure data which are error free corresponding to known O.D.F.s) have given excellent results (Dahms and Bunge 1988; Wagner and Dahms 1991). For real textures the situation is somewhat different: usually the iterative procedure converges after $n$ steps to a solution, denoted from now on $f_{n}(g)$, which still contains some small negative values. Qualitatively one can assess that the more important the errors in the experimental starting pole figures the more important the negative values in the calculated O.D.F. $f_{n}(g)$. 


\section{FINAL CORRECTION}

There are two points of view regarding such a situation. The first one consists in accepting the calculated O.D.F.: $f_{n}(g)$ is the best approximation which can be obtained with data containing errors and the amount of negative values in $f_{n}(g)$ represents some "measure" of these errors. In the second one the solution $f_{n}(g)$ is not accepted just as it is because negative values have no physical meaning and a correction will be applied in order to get a final solution, ${ }^{F} f(g)$, which is strictly positive. Obviously this correction will require to modify the even part of the O.D.F., $\widetilde{f}(g)$, which implies that the recalculated pole figures will be modified and will no more be the closest (in the sense of least square) to the experimental ones. The methods proposed by Van Houtte (quadratic method (Van Houtte, 1983), exponential method (Van Houtte, 1991)) support in some manner this second point of view.

In the iterative positivity technique there is an easy way to perform such a final correction. Assuming that the positivity technique has converged towards a solution $f_{n}(g)$ which still contains negative values a strictly positive solution, $F(g)$, can be obtained by:

$$
{ }^{F} f(g)=k\left(f_{n}(g)+{ }^{F} \hat{f}(g)\right)
$$

where $F \hat{f}(g)$, similarly as in (2), is defined by

$$
F \hat{f}(g)=\left\{\begin{array}{lll}
0 & \text { if } & f_{n}(g) \geqslant 0 \\
-f_{n}(g) & \text { if } & f_{n}(g)<0
\end{array}\right.
$$

and where $k$ is a normalization factor which ensures that

$$
\oint^{F} f(g) \mathrm{d} g=1
$$

If $\alpha$ is the integral over the whole Euler space of the function ${ }^{F} \hat{f}(g), k$ reads:

$$
k=1 /(1+\alpha)
$$

\section{CONCLUDING REMARKS}

The first point is that formula (3) implies small modifications of $f_{n}(g)$ as long as pole figure measurements which are the input data of the problem are of rather good quality. Typically $\alpha$, the integral of $F \hat{f}(g)$, is in the range 0 . to 0.1 . It should also be remarked that formula (3) is not derived from mathematical considerations but is deduced from the fact that negative values have no physical meaning and that the closest acceptable values are zero.

Demonstrating the efficiency of the proposed correction is somewhat difficult. In the case of real textures with $f_{n}(g)$ containing negative values one cannot know if the final correction leads to a more precise solution because the true O.D.F. is unknown. If model pole figures are considered $f_{n}(g)$ does not exhibit negative values and obviously no more correction is necessary. The only way to test the improvement supplied by relation (3) consists in the following scheme of calculation: starting from a model O.D.F. several corresponding pole figures are calculated. They are then deliberately falsified by simulating for example 
statistical errors or background correction errors. Such data can then be used for texture analysis and the result with or without final correction can be compared with the known true O.D.F. Such calculations will be presented in a next paper (Wagner, in preparation).

\section{References}

Van Houtte, P. (1983) Textures and Microstructures 6, p. 1-20.

Dahms, M. and Bunge, H. J. (1988). Textures and Microstructures 10, p. 21-35.

Dahms, M. and Bunge, H. J. (1989). J. Appl. Cryst. 22, p. 439.

Wagner, F., Humbert, M., Muller, J. and Esling, C. (1990). Europhys. Lett. 11, p. 479-483.

Wagner, F. and Dahms, M. (1991). In Advances and Applications of Quantitative Texture Analysis ed. H. J. Bunge and C. Esling, in print.

Wagner, F., Humbert, M., Esling, C. and Muller, J. (1991). Proceedings of the ICOTOM 9, in print. Van Houtte, P. (1991). Textures and Microstructures in print.

Wagner, F. Influence of errors in pole figures on the O.D.F. calculation, in preparation. 